\title{
Fabrication of durable fluorine-free superhydrophobic polyethersulfone (PES) composite coating enhanced by assembled MMT-SiO $\mathrm{Manoparticles}_{2}$
}

Xiguang Zhang, Huaiyuan Wang ${ }^{*}$, Zhanjian Liu, Yixing Zhu, Shiqi Wu, Chijia Wang, Yanji Zhu

College of Chemistry and Chemical Engineering, Northeast Petroleum University, Daqing, 163318, Republic of China

\begin{abstract}
A durable fluorine-free polyethersulfone (PES) superhydrophobic composite coating with excellent wear-resistant and anti-corrosion properties has been successfully fabricated by combining sol-gel and spray technology. The robust micro/nano-structures of the prepared surface were established by introducing binary montmorillonite-silica $\left(\mathrm{MMT}-\mathrm{SiO}_{2}\right)$ assembled composite particles, which were formed by in-situ growth of $\mathrm{SiO}_{2}$ on MMT surfaces via sol-gel. Combined with the low surface energy of amino silicon oil (APDMS), the fluorine-free superhydrophoic PES coating was obtained with high water contact angle $156.1 \pm 1.1^{\circ}$ and low sliding angle $4.8 \pm 0.7^{\circ}$.

The anti-wear of the final PES/APDMS/MMT-SiO ${ }_{2}$ superhydrophobic coating can reach up to 60100 cycles, which is outdistancing the pure PES coating (6800 cycles) and the $\mathrm{PES} / \mathrm{MMT} / \mathrm{SiO}_{2}$ coating prepared by simple physical mixture
\end{abstract}

"Corresponding authors. Fax: +86 459 6503083. E-mail addresses: wanghyjiji@ 163.com 
(18200 cycles). The enhanced wear resistance property can be mainly attributed to the lubrication performance of APDMS and stable interface bonding force between the MMT surface and $\mathrm{SiO}_{2}$. Simultaneously, potentiodynamic polarization curves and electrochemical impedance spectroscopy exhibited the outstanding anti-corrosion property of PES/APDMS/MMT-SiO 2 composite coating, with low corrosion current $\left(1.6 \times 10^{-10} \mathrm{~A} / \mathrm{cm}^{2}\right)$ and high protection efficiency (99.999\%) even after $30 \mathrm{~d}$ immersion process. These test results show that this durable superhydrophobic PES composite coating can be hopefully to provide the possibility of industrial application.

Key words: superhydrophobic; polyethersulfone; wear-resistance; corrosion resistance; fluorine-free.

\section{Introduction}

Inspired by the self-cleaning lotus leaves $[1,2]$, superhydrophobic surfaces with high water contact angles (WCAs $>150^{\circ}$ ) and low water sliding angles (WSAs $<10^{\circ}$ ) have attracted more attention from science researchers due to its outstanding performances, such as self-cleaning [3], anti-ice [4], anti-fouling [5] and anti-drag [6]. With the in-depth study, researchers found that low surface energy materials and micro/nano binary structure are the two essential conditions for a superhydrophobic surface $[7,8]$. On the basis of this theory, fluorochemicals as the lowest surface energy materials have been widely used to fabricate superhydrophobic surfaces in recent years. For instance, Lin et al. fabricated a polydimethylsiloxane (PDMS)/ fluorinated alkyl silane (FAS) superhydrophobic surface by introducing FAS 
functionalised silica nanoparticles on fabrics [9]. Li et al. fabricated a superhydrophobic flower-like $\mathrm{CuO}$ film on the surface of X90 pipeline steel by controlling the solution-immersion process and modifying with perfluorooctanoic acid (PFOA) [10]. Despite all this, the application of fluorochemicals has been limited under the consideration of the expensive price and potential adverse effects on human offspring $[11,12]$.

In recent years, the long carbon chain compounds and silicon-oxygen polymers as the fluorine-free low surface energy materials have attracted wide attention because of the low-cost, nontoxic and environment friendly properties [13]. For instance, Yu et al. fabricated superhydrophobic aluminum surfaces with corrosion resistance by modifying the chemical etching surface with stearic acid [14]. Yeh et al. reported a superhydrophobic PDMS surface with Xanthosoma sagittifolium leaf morphology by soft template method [15]. Although some fluorine-free superhydrophobic coatings have been developed, the complex preparation and weakly mechanical stability of these coatings have seriously compromised their scale-up manufacturing and applications [16]. Therefore, it is still a great challenge to prepare durable fluorine-free superhydrophobic surfaces for industrial applications.

Polyethersulfone (PES) as a high-performance engineering material has excellent flame resistance, mechanical stability and chemical inertness properties [17-19]. Recent studies demonstrated that PES was mainly used to fabricate high-flux membranes and mechanical components rather than using in preparing coatings $[20$, 21]. Understanding the intrinsic hydrophobicity and wearability of PES, it can be 
hopefully used to fabricate a durable fluorine-free superhydrophobic coating [22]. Thus, the unique wear-resistance and chemical inertness properties of PES have greater advantages to broaden the range of potential applications of the prepared PES superhydrophobic surfaces in the high-temperature operation or wear-resistance fields, such as heat exchanger, internal pipeline coating and submersible pump surface. In addition, we introduced amino silicon oil (APDMS), a fluorine-free and safe material, to further reduce the PES surface energy by its large amounts of -Si-O- groups. Compared with the common polydimethylsiloxane (PDMS), extra amino groups can provide more possible for interface modification [23]. To enhance the mechanical properties of the coating, montmorillonite (MMT) and nano-SiO ${ }_{2}$ with special micron lamellar and spheroidal structures particles captured our attention. The special micron lamellar of MMT can effectively enhance the anti-corrosion performance of coatings due to the intercalating effect. The introduction of nano- $\mathrm{SiO}_{2}$ into coatings makes it possible to satisfy the structure requirement of superhydrophobic coatings. In order to enhance the interfacial interaction between nano- $\mathrm{SiO}_{2}$ and $\mathrm{MMT}$, nano- $\mathrm{SiO}_{2}$ was in-situ growth on the surface of MMT by sol-gel. Furthermore, the nano/micro-structures of coating can also be established by introducing of $\mathrm{MMT}-\mathrm{SiO}_{2}$ particles.

In this work, we successfully prepared a fluorine-free superhydrophobic PES/APDMS/MMT-SiO 2 coating with wear/corrosion resistance by the combination of sol-gel and spraying technique. The coating shows excellent superhydrophobicity with WCA of $156.1 \pm 1.1^{\circ}$ and SA of $4.8 \pm 0.7^{\circ}$. The superhydrophobic 
PES/APDMS/MMT-SiO ${ }_{2}$ coating exhibits outstanding wear-resistant property, which is nearly ten times more than PES coating. The corrosion-resistance property of the prepared coating was investigated in detail. It is believed that the prepared superhydrophobic PES/APDMS/MMT- $\mathrm{SiO}_{2}$ coating demonstrated great developmental potential in practical application.

\section{Experimental}

\subsection{Materials}

Polyethersulfone (PES) powder with average diameter of $500 \mathrm{~nm}$ was purchased from DuPont (USA). Montmorillonite (MMT) powder with average diameter of 2-5 $\mu \mathrm{m}$ was offered by Shenzhen Haicheng Co. Ltd (China). Tetraethylorthosilicate (TEOS) was provided by Aladdin. The absolute ethanol (purity $\geq 99.8$ wt.\%) and ammonium hydroxide $\left(\mathrm{NH}_{3} \cdot \mathrm{H}_{2} \mathrm{O}\right)$ were supplied by Huadong Reagent Factory, Shenyang (China). Amino Silicon Oil (APDMS) was bought from Jinan Guobang chemical Co. Ltd (China).

\subsection{Fabrication of PES/APDMS/MMT-SiO ${ }_{2}$ superhydrophobic coating}

Preparation process of the superhydrophobic PES/APDMS/MMT-SiO ${ }_{2}$ coating was shown in Fig 1. The specific steps are shown below: First, the aluminum plate (1100 grade, $80 \mathrm{~mm} \times 80 \mathrm{~mm} \times 1 \mathrm{~mm}$ ) was polished with 800 mesh sand papers in a certain direction, and then ultrasonically in absolute alcohol to remove surface impurities. Afterwards, the substrate was etched by hydrochloric acid solution (2.5 $\mathrm{mol} / \mathrm{L}$ ) for $30 \mathrm{~min}$ and rinsed extensively with deionized water. Compared with the 
original smooth surface (Fig.1 inset SEM (a)), micro-structures were obtained on the surface of aluminum substrate (Fig.1 inset SEM (b)). In addition, some uniform and tiny nano-structures can be achieved on the micro-structure surface by boiling the etched substrate in water for 25 min (Fig.1 inset SEM (c)). These special multilevel structures with massive hydroxyl groups can enhance the adhesion between the composite coating and aluminum substrate.

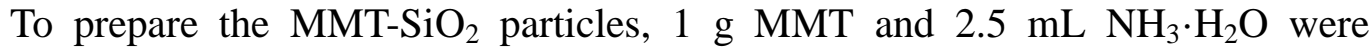
ultrasonically dispersed in $50 \mathrm{~mL}$ absolute ethanol to form a uniform solution. $2.5 \mathrm{ml}$ of TEOS was diluted with $10 \mathrm{ml}$ of absolute ethanol and then added into the above 50 $\mathrm{ml}$ of MMT ethanol solution in $10 \mathrm{~min}$. After $6 \mathrm{~h}$ hydrolization of TEOS under magnetic stirring, the $\mathrm{MMT}-\mathrm{SiO}_{2}$ particles were obtained by grafting nano- $\mathrm{SiO}_{2}$ on the MMT surfaces. Subsequently, $1.5 \mathrm{~g}$ PES powder, $0.03 \mathrm{~g}$ APDMS and $0.03 \mathrm{~g}$ MMT-SiO ${ }_{2}$ were added into $10 \mathrm{ml}$ ethanol solution to form a uniform solution under ultrasonic stirring for $20 \mathrm{~min}$. The underlying layer of the coating was prepared by spraying the above solution on the as-prepared aluminum surface under a pressure of 3 bar with 5 min spraying. The distance between the substrate and gun is $20 \mathrm{~cm}$ and the type of gas we used is air. The underlying layer was obtained after calcined at $240^{\circ} \mathrm{C}$ for $1 \mathrm{~h}$. In order to optimize the performance of the coating, $0.1 \mathrm{~g}$ APDMS, 1.0 g PES and different amounts of $\mathrm{MMT}^{-\mathrm{SiO}_{2}}$ composite particles were mixed into 10 $\mathrm{ml}$ ethanol for the superficial coating. The mixture was sprayed onto the underlying coating with a pressure of 6 bar. Finally, the composite coatings with internal nano/micro-structures (Fig. 1 inset SEM (d)) were obtained after calcined at $240^{\circ} \mathrm{C}$ for 
$1.5 \mathrm{~h}$.

\subsection{Characterization}

The water contact angles (WCAs) of the prepared coating were measured by Contact Angle Meter (JGW-360A, Chengdeshi Shipeng Detection Equipment Co. Ltd.) with approximately $5 \mu \mathrm{L}$ distilled water droplets. The reported values were the average of 5 measurements made on different areas of the coating surface. The morphology of the prepared coating and the in-situ growth status of the $\mathrm{SiO}_{2}$ on the MMT surface were obtained by scanning electron microscopy (SEM) (Quanta 200). Reactions between the materials and the chemical composition were confirmed by Tensor-27 infrared spectrometer (FT-IR). The thickness of the prepared coatings was measured by Coating Thickness Gauge (SaluTron ComBi-D3, Germany). To ensure the authenticity of the data, the thickness for each sample was obtained from the average measurement of five different areas. The wear resistance was evaluated by Taber wear and abrasion testers (JST-3393). And the coating is defined as a failure at the moment when any area of the substrate was exposed to ambient air. The corrosion resistance of the composite coating was evaluated using an electrochemical workstation (Autolab PGSTAT302N) at room temperature in $3.5 \mathrm{wt} . \% \mathrm{NaCl}$.

\section{Results and discussion}

\subsection{Analysis of the in-situ growth ${\mathrm{MMT}-\mathrm{SiO}_{2} \text { particles }}$}

The structural unit of MMT is composed of an alumina octahedral sheet sandwiched between two silica tetrahedral sheets. Thus, the surface of MMT has free hydroxyl groups, such as -Al-O- $\left(572 \mathrm{~cm}^{-1}\right)$ and -Si-O- $\left(823 \mathrm{~cm}^{-1}\right)$ (Fig. 2a (i)) [24], 
which can be further functionalized by surface grafting. During Stöber method, the nano- $\mathrm{SiO}_{2}$ particles can be in-situ grafted on MMT surface by chemical bonding. As shown in Fig. 2b, the hydrolysis of TEOS in the presence of the ammonia catalyst can form nano- $\mathrm{SiO}_{2}$ precursor [25]. The nano- $\mathrm{SiO}_{2}$ can be effectively grafted on the two-dimensional (2D) MMT platelets though the condensation reaction between $\mathrm{Si}-\mathrm{OH}$ and $\mathrm{Al}-\mathrm{OH}[26]$.

The presence of the condensation reaction was further confirmed by the FT-IR spectra (Fig. 2a). Compared with the spectrum of MMT, the peak of $\mathrm{MMT}^{-\mathrm{SiO}_{2}}$ shifted from $823 \mathrm{~cm}^{-1}$ to $861 \mathrm{~cm}^{-1}$ due to the formation of the stable hydrogen bonding between $\mathrm{SiO}_{2}$ and MMT surface. The asymmetric stretching vibration peak of $\mathrm{Si}-\mathrm{O}-\mathrm{Si}$ at $1079 \mathrm{~cm}^{-1}$ changed narrow and sharp with the formation of silica (Fig. 2a) [27]. As shown in Fig. 2a (ii), some new peaks emerged at $951 \mathrm{~cm}^{-1}$ and $1385 \mathrm{~cm}^{-1}$, which correspond to the stretching mode $\mathrm{Si}-\mathrm{OH}$ typical of gel structure and the organic residues $(\mathrm{C}-\mathrm{H})$ of sol-gel, respectively [26, 28]. Thus, FT-IR can confirm the in-situ growth of nano-SiO 2 on MMT surface during sol-gel.

In addition, the in-situ growth of nano- $\mathrm{SiO}_{2}$ on MMT surface can also be effectively proved by SEM analysis. Fig. 2 shows the surface morphologies in different magnifications of the original MMT (c1, c2), the prepared $\mathrm{MMT}^{-\mathrm{SiO}_{2}}$ particles $(\mathrm{d} 1, \mathrm{~d} 2)$ and the MMT physically mixed with nano-SiO${ }_{2}(\mathrm{e} 1, \mathrm{e} 2)$. Compared with the smooth MMT surface (Fig. 2 (c1)), the compact nano/micro structure was formed on MMT surface with close-grained nano-SiO $\mathrm{S}_{2}$ after 6 h hydrolysis of TEOS (Fig. $2(\mathrm{~d} 1, \mathrm{~d} 2))$. In contrast, the SEM morphology of the physical mixture of 
nano- $\mathrm{SiO}_{2}$ and MMT was distinguished clearly without any interface bonding force (Fig. 2 (e1)). In addition, loose and porous nano-SiO 2 shows in Fig. 2 (e2) is unfavorable for the mechanical durability of the prepared coating. The strong interfacial interation provided by the in-situ grown $\mathrm{SiO}_{2}$ on the MMT can remarkly enhance the mechanical durability of the prepared coating. Moreover, the compact nano/micro structures are crucial for the superhydrophobicity of the prepared coating. Combined with the low surface energy provided by APDMS, the superhydrophobicity of the prepared coating can be achieved.

\subsection{Surface wettability analysis}

As shown in Fig. $3(a, b)$, the influence of addition different amount of the pure MMT and MMT-SiO ${ }_{2}$ on the wettabilities of the prepared coatings was investigated under a certain amount of APDMS. It can be clearly seen that the hydrophobic ability of the composite coating was enhanced by increasing the content of particles. However, the PES/APDMS/MMT coating with 16 wt.\% particles still cannot achieve superhydrophobicity $\left(\mathrm{WCA}=143 \pm 1.2^{\circ}, \mathrm{SA}=27 \pm 1.7^{\circ}\right)$ (Fig. $\left.3 \mathrm{a}\right)$.

To optimize the surface wettabilities, the as-prepared $\mathrm{MMT}^{-\mathrm{SiO}_{2}}$ as the filling particles instead of MMT were added into the composite coating. Due to the fact that adhesion performance of coating will be reduced by the increasing content of particle, the coating with 12 wt.\% $\mathrm{MMT}^{-\mathrm{SiO}_{2}}$ (named as PES/APDMS/MMT-SiO${ }_{2}-12$ ) as the optimum superhydrophobic surface can be achieved with WCA of $156.1 \pm 1.1^{\circ}$ and SA of $4.8 \pm 0.7^{\circ}$ (Fig. 3b). The reason for the differences in wettability is that the in-situ growth of nano- $\mathrm{SiO}_{2}$ on MMT surface with large specific surface area can reduced the 
solid-liquid contact area and capture much more gas than pure MMT (Fig. 3c) [29].

Simultaneously, the prepared coatings with different content of APDMS also exhibit an increasing trend on wettability under filling a certain amount of particles, as shown in Fig. 3d. Nonetheless, the wettability of the PES/APDMS/MMT-SiO ${ }_{2}$ coating won't be moved continuously upward by further increasing the content of APDMS more than $0.1 \mathrm{~g}$. Therefore, the optimal superhydrophobic surface can be obtained by adding 12 wt.\% MMT-SiO 2 and modifying with $0.1 \mathrm{~g}$ APDMS.

\subsection{Surface morphologies analysis}

Fig. 4 shows the SEM images of the prepared coatings with 12 wt.\% MMT, 6 wt.\% and 12 wt.\% MMT-SiO 2 under different magnification. From Fig. 4 (a1, a2), we can observe that the morphology of the prepared coating with just $12 \mathrm{wt} . \%$ MMT was the relatively smooth surface except a sparse distribution of surface embossment. With the addition of $6 \mathrm{wt} . \% \mathrm{MMT}-\mathrm{SiO}_{2}$, a small amount of air holes and hierarchical structures were formed on the coating surface, as shown in Fig. 4 (b1, b2). By extending the addition of $\mathrm{MMT}_{-} \mathrm{SiO}_{2}$ to $12 \mathrm{wt} . \%$, the surface morphologies of the PES/APDMS/MMT-SiO 2 -12 coating exhibited both some micro/nano-pores and small mastoids (Fig. 4 c1). The inserted SEM image with high magnification demonstrated that the mastoid was constituted by amount of fragments, like a "flower cluster".

From Fig. 4 c2, it can be clearly seen that there are numerous nano mastoids (about $200 \mathrm{~nm}$ ) on the walls of the pores, which is similar to "coralline" morphology. The reason for the significant morphological changes of the prepared coating can be mainly attributed to the in-situ growth of nano-SiO 2 , forming porous nano/micro 
structure on MMT surface. It is known that porous structure has strong adsorption of solvent [30]. During the heat treatment, the solvent adsorbed by $\mathrm{MMT}^{-\mathrm{SiO}_{2}}$ can act as pore-forming agent to form the complicated hierarchical morphology in the coating when the vaporization occurred. It indicated that the addition of $\mathrm{MMT}_{-} \mathrm{SiO}_{2}$ has great influence on the surface morphology of the prepared coating, proving the essential role of MMT-SiO $\mathrm{M}_{2}$ for the prepared superhydrophobic coating.

\subsection{Wear resistance of the coatings}

The friction tests of the prepared coatings were conducted on Taber Wear Testing Machine, which comprises $500 \mathrm{~g}$ loading wheel and friction wheel covered with 1000-mash sandpaper under $50 \mathrm{kPa}$ loading and a sliding speed of $0.14 \mathrm{~m} / \mathrm{s}$. And, the thicknesses of the pure PES, PES/MMT/SiO $2, \mathrm{PES} / \mathrm{MMT}-\mathrm{SiO}_{2}$ and superhydrophobic PES/APDMS/MMT-SiO 2 coatings are $79.8 \mu \mathrm{m}, 82.1 \mu \mathrm{m}, 80.9 \mu \mathrm{m}$ and $81.6 \mu \mathrm{m}$, respectively. As shown in Fig. 5a, the wear life of the pure PES coating was only 6800 cycles. With the addition of 12 wt.\% $\mathrm{MMT} / \mathrm{SiO}_{2}$ (physical mixing), the wear-resistance ability of the $\mathrm{PES} / \mathrm{MMT} / \mathrm{SiO}_{2}$ coating was improved to 18200 cycles. The enhanced wear-resistance by addition of inorganic fillers was mainly attributable to the improvement of the coating hardness, which is proportional to the wear-resistance ability [31].

Compared with the PES/MMT/SiO${ }_{2}$ coating, the wear life of the PES/MMT-SiO can withstand much longer time of 31700 cycles. The reason for the further enhanced wear-resistance can be ascribed to the in-situ growth of nano-SiO${ }_{2}$ and MMT surface, increasing the interface stability between nano- $\mathrm{SiO}_{2}$ and MMT surface. Moreover, the 
designed porous nano/micro-structures of the prepared coating, like a "warehouse", can capture debris of the coating to increase the wear life of the prepared composite coating. With the addition of a small amount of APDMS, the wear life of the final superhydrophobic PES/APDMS/MMT-SiO 2 coating was recorded to be the longest time of 60100 cycles. The longest wear life of the PES/APDMS/MMT-SiO ${ }_{2}$ coating can be mainly attributed to the self-lubrication of APDMS and $\mathrm{MMT}^{-\mathrm{SiO}_{2}}$ hybrid assembly [32].

The PES/APDMS/MMT-SiO 2 coating also demonstrated long-term durable superhydrophobicity even after 1000 cycles of rubbing cycles. The WCA of the rubbed coating decreased slightly from $156 \pm 1.1^{\circ}$ to $155 \pm 1.3^{\circ}$, indicating the superhydrophobic durability (Fig. 5b). It can be attributed to the existence of the new micro/nano structure consisted of abrasive dust and abrasive scratch on the rubbed surface (Fig. 5c). After 6000 times of rubbing cycles, the WCA of the rubbed coating can still maintain $127 \pm 1.2^{\circ}$. Interestingly, the superhydrophobicity can be restored with WCA back to $154 \pm 1.2^{\circ}$ by simply surface treated with 600 mesh sandpaper. The restored superhydrophobicity of the rubbed coating can be explained by the new formed "whisker"-like micro/nano structures after surface roughening (Fig. 5d). Therefore, the test results indicated that the prepared PES/APDMS/MMT-SiO coating can not only withstand long-time abrasion but also recover its superhydrophobicity after surface roughening. 


\subsection{Corrosion resistance of the coatings}

Table 1. Polarization parameters values of different samples.

\begin{tabular}{|c|c|c|c|c|c|}
\hline Sample & $\begin{array}{c}\text { Immersion } \\
\text { time }\end{array}$ & $\begin{array}{l}\mathbf{E}_{\text {corr }} \\
(\mathrm{mV})\end{array}$ & $\begin{array}{c}\mathbf{I}_{\text {corr }} \\
\left(\mathrm{A} / \mathrm{cm}^{2}\right)\end{array}$ & $\begin{array}{c}\text { C.R. } \\
(\mu \mathrm{m} / \text { year })\end{array}$ & $\begin{array}{l}\text { P.E. } \\
(\%)\end{array}$ \\
\hline Uncoated & $24 \mathrm{~h}(1 \mathrm{~d})$ & -777 & $1.1 \times 10^{-5}$ & 130 & - \\
\hline PES & $720 \mathrm{~h}(30 \mathrm{~d})$ & -552 & $1.5 \times 10^{-8}$ & 0.183 & 99.864 \\
\hline PES/APDMS/MMT-SiO & $720 \mathrm{~h}(30 \mathrm{~d})$ & -285 & $1.6 \times 10^{-10}$ & 0.002 & 99.999 \\
\hline
\end{tabular}

Fig. 6 (A) shows the potentiodynamic polarization curves (PPC) of the pure aluminum plate, PES coating and the superhydrophobic PES/APDMS/MMT-SiO ${ }_{2}$ coating immersed in $3.5 \mathrm{wt} . \% \mathrm{NaCl}$ solution for $1 \mathrm{~d}, 30 \mathrm{~d}$ and $30 \mathrm{~d}$, respectively. The corresponding polarization parameters were summarized in Table 1. Due to the excellent shielding effect, the potentiodynamic polarization curves of PES coating and the superhydrophobic PES/APDMS/MMT-SiO 2 coating can be obtained until after 30 d immersion treated. From Fig. 6 (A), it can be seen that the $E_{\text {corr }}$ and $I_{\text {corr }}$ of the aluminum plate coated with PES present positive movement from $-777 \mathrm{mV}$ and $1.1 \times 10^{-5} \mathrm{~A} / \mathrm{cm}^{2}$ to $-552 \mathrm{mV}$ and $1.5 \times 10^{-8} \mathrm{~A} / \mathrm{cm}^{2}$ as compared to the pure aluminum plate.

It is well known that a lower corrosion current density ( $\left.\mathrm{I}_{\text {corr }}\right)$ or a higher corrosion potential $\left(\mathrm{E}_{\mathrm{corr}}\right)$ is a typical representation of the lower corrosion rate and the better corrosion resistance [33]. Therefore, the test result demonstrated that the corrosion-resistant performance of the PES coating is better than that of the pure aluminum plate. The enhanced anti-corrosion protection of aluminum coated with PES is mainly due to the physical shielding effect of the PES coating surface, which 
can prevent the aluminum plate from the directly contacting with corrosive liquid (Fig. $6(\mathrm{~B}, \mathrm{a}, \mathrm{b}))$. In the case of the superhydrophobic PES/APDMS/MMT-SiO ${ }_{2}$ coating, the corrosion potential $\left(\mathrm{E}_{\text {corr }}\right)$ and corrosion current $\left(\mathrm{I}_{\text {corr }}\right)$ show a marked positive shift to $-258 \mathrm{mV}$ and $1.6 \times 10^{-10} \mathrm{~A} / \mathrm{cm}^{2}$ as compared to those of the pure aluminum plate and the PES coating, indicting the excellent corrosion-resistant performance.

Moreover, corrosion rate (C.R.) and protection efficiency (P.E.) can be directly used to evaluate the anti-corrosion property for each sample. As shown in Table 1, the corrosion rate of the superhydrophobic PES/APDMS/MMT-SiO ${ }_{2}$ coating $(0.002$ $\mu \mathrm{m} /$ year $)$ is about 2 orders of magnitude lower than the PES $(0.183 \mu \mathrm{m} /$ year $)$, not to mention the uncoated aluminum plate $(130 \mu \mathrm{m} /$ year $)$.

The corrosion protection efficiency (P.E.) can be obtained from the measured $\mathrm{I}_{\text {corr }}$ values according to eq. 1 [34].

$$
\text { P.E. }(\%)=\left[1-\frac{I_{\text {corr }}^{c}}{I_{\text {corr }}^{o}}\right] \times 100
$$

Where $I_{\text {corr }}^{c}$ and $I_{\text {corr }}^{o}$ are the corrosion current of coated Al substrate and bare Al substrate, respectively.

The calculations indicate that the superhydrophobic PES/APDMS/MMT-SiO ${ }_{2}$ coating possess the optimal corrosion-resistant performance with P.E. (99.999\%) even after $30 \mathrm{~d}$ immersion. This can be ascribed to the micro/nano-structure designed by MMT-SiO ${ }_{2}$ composite particles, which can trap more air. The physical shielding effect of the superhydrophobic PES/APDMS/MMT-SiO ${ }_{2}$ coating was further enhanced by the superficial air layer, leading to the improvement of the corrosion-resistant performance. These air can prevent permeate of the corrosion liquid and other species 
such as $\mathrm{Cl}^{-}$and the $\mathrm{O}_{2}$.

To investigate the anticorrosion mechanism of the composite coatings, the samples were immersed in $3.5 \mathrm{wt} \% \mathrm{NaCl}$ solution and tested through electrochemical impedance spectroscopy (EIS) after $1 \mathrm{~d}, 7 \mathrm{~d}, 15 \mathrm{~d}, 30 \mathrm{~d}, 60 \mathrm{~d}$. The Nyquist spectrum of bare $\mathrm{Al}$ substrate shows a semicircle-like shape after 1 day immersion (Fig.7 a). In addition, one time constant exhibited at around $10 \mathrm{~Hz}$ in the Bode plot (Fig.7 c), which confirmed that the corrosion process occurred at the aluminum surface. The process generally attributed to the adsorbed species resulting from the $\mathrm{Al}$ dissolution and the adsorption of chloride ions [35].

The impedance spectra of the pure PES coating were obtained after different immersion time, as shown in Fig. 7(d, e, f). With the increased soak time from $1 \mathrm{~d}$ to $60 \mathrm{~d}$, the semicircle diameters in Nyquist plot gradually become smaller (Fig. 7d). A capacitive loop tail was appeared at low frequencies since $7 \mathrm{~d}$ immersion and the locations of the turning points moved forward gradually with the increasing time to 15 $\mathrm{d}, 30 \mathrm{~d}$ and $60 \mathrm{~d}$. In the Bode plots, low-frequency impedance modulus value $\left(|\mathrm{Z}|_{0.1 \mathrm{~Hz}}\right)$ fell by around $95 \%$ and the phase angles decrease to about -15 degree after 60 days (Fig. 7(e, f)). These changes indicated that the corrosion protection gradually decreased with extending soak time due to the increase capacitance caused by water uptake in the coating [36].

Compared with bare Al substrate and pure PES coating, the Nyquist spectra of final superhydrophobic coating shows larger semicircle diameter, corresponding to a higher corrosion property and a lower corrosion rate (Fig. 7g) [37]. In Bode plots, 
overlapping straight lines are shown in Fig. 7h with a high $|\mathrm{Z}|_{0.1 \mathrm{~Hz}}$ that reaches to $1.7 \times 10^{8} \mathrm{~cm}^{2}$ even after 60 days of immersion. Furthermore, the phase angles in Fig. 7i were only slightly decreased in the whole frequency range over the whole immersion time. These significant improvements were contributed to the air dielectric film trapped in micro-nano scale surface. The air film, acted as a pure parallel plate capacitor, can inhibit the electrolyte solution penetrated into organic coating and prevent the electron transfer between the electrolyte and the substrate. Thus, the final superhydrophobic PES/APDMS/MMT-SiO ${ }_{2}$ coating shows the optimal corrosion resistant performance.

\section{Conclusions}

In summary, a durable fluorine-free superhydrophobic PES coating enhanced by assembled $\mathrm{MMT}-\mathrm{SiO}_{2}$ nanoparticles was successfully fabricated by spaying technology. Some detail conclusions were shown as follow:

1. The hydrophobic ability of PES coating surface improved by the addition of MMT-SiO ${ }_{2}$ assembled composite particles and APDMS incorporation. 12 wt.\% MMT-SiO ${ }_{2}$ and $0.1 \mathrm{~g}$ APDMS as the optimum addition to obtained the best superhydrophobic ability $\left(\mathrm{WCA}=156 \pm 1.1^{\circ}, \mathrm{SA}=4.5 \pm 0.7^{\circ}\right)$.

2. Compared with physical mixing of $\mathrm{MMT}$ and $\mathrm{SiO}_{2}$, the in-situ $\mathrm{MMT}-\mathrm{SiO}_{2}$ composite particles enhanced the interface force within PES coating. And anti-wear resistance of the in-situ $\mathrm{MMT}_{-} \mathrm{SiO}_{2}$ reinforced PES coating is 3.3 times higher than that of PES coating with physical mixing of MMT and $\mathrm{SiO}_{2}$.

3. The in-situ MMT-SiO ${ }_{2}$ reinforced PES composite coating exhibited excellent 
corrosion protection ability, which is about 2 orders of magnitude higher than the PES.

\section{Acknowledgments}

The research is financially supported by the National Young Top Talents Plan of

China (2013042), National Science Foundation of China (Grant No. 51175066, 21676052, 21606042), the Science Foundation for Distinguished Young Scholars of Heilongjiang Province (JC201403), and Graduate student innovation project (YJSCX2015-019NEPU, YJSCX2016-016NEPU).

\section{References}

[1] J. Zhang, L. Wu, B. Li, L. Li, S. Seeger, A. Wang, Evaporation-induced transition from nepenthes pitcher-inspired slippery surfaces to lotus leaf-inspired superoleophobic surfaces, Langmuir 30 (2014) 14292-14299.

[2] H. Zhu, Z. Guo, Hybrid engineered materials with high water-collecting efficiency inspired by Namib Desert beetles, Chem. Commun. 52 (2016) 6809-6812.

[3] Y. Lu, S. Sathasivam, J. Song, C.R. Crick, C.J. Carmalt, I.P. Parkin, Robust self-cleaning surfaces that function when exposed to either air or oil, Science 347 (2015) 1132-1135.

[4] P. Guo, M. Wen, L. Wang, Y. Zheng, Strong anti-ice ability of nanohairs over micro-ratchet structures, Nanoscale 6 (2014) 3917-3920.

[5] X. Lu, Y. Peng, L. Ge, R. Lin, Z. Zhu, S. Liu, Amphiphobic PVDF composite membranes for anti-fouling direct contact membrane distillation, J. Membrane Sci. 505 (2016) 61-69. 
[6] J. Hu, W. Yuan, Z. Yan, B. Zhou, Y. Tang, Z. Li, Fabricating an enhanced stable superhydrophobic surface on copper plates by introducing a sintering process, Appl. Surf. Sci. 355 (2015) 145-152.

[7] X. Zhang, Z. Li, K. Liu, L. Jiang, Bioinspired Multifunctional Foam with Self Cleaning and Oil/Water Separation, Adv. Funct. Mater. 23 (2013) 2881-2886.

[8] B. Bhushan, Y.C. Jung, Natural and biomimetic artificial surfaces for superhydrophobicity, self-cleaning, low adhesion, and drag reduction, Prog. Mater. Sci. 56 (2011) 1-108.

[9] H. Zhou, H. Wang, H. Niu, A. Gestos, X. Wang, T. Lin, Fluoroalkyl silane modified silicone rubber/nanoparticle composite: a super durable, robust superhydrophobic fabric coating, Adv. Mater. 24 (2012) 2409-2412.

[10] H. Li, S. Yu, X. Han, Fabrication of CuO hierarchical flower-like structures with biomimetic superamphiphobic, self-cleaning and corrosion resistance properties, Chem. Eng. J. 283 (2016) 1443-1454.

[11] T.M. Schutzius, I.S. Bayer, J. Qin, D. Waldroup, C.M. Megaridis, Water-based, nonfluorinated dispersions for environmentally benign, large-area, superhydrophobic coatings, ACS Appl. Mater. Inter. 5 (2013) 13419-13425.

[12] C. Holtzinger, B. Niparte, S. Wächter, G. Berthomé, D. Riassetto, M. Langlet, Superhydrophobic TiO 2 coatings formed through a non-fluorinated wet chemistry route, Surf. Sci. 617 (2013) 141-148.

[13] Y. Li, X. Men, X. Zhu, B. Ge, F. Chu, Z. Zhang, One-step spraying to fabricate nonfluorinated superhydrophobic coatings with high transparency, J. Mater. Sci. 
51 (2016) 2411-2419.

[14] D. Zang, R. Zhu, W. Zhang, J. Wu, X. Yu, Y. Zhang, Stearic acid modified aluminum surfaces with controlled wetting properties and corrosion resistance, Corros. Sci. 83 (2014) 86-93.

[15] C.-W. Peng, K.-C. Chang, C.-J. Weng, M.-C. Lai, C.-H. Hsu, S.-C. Hsu, S.-Y. Li, Y. Wei, J.-M. Yeh, UV-curable nanocasting technique to prepare bio-mimetic super-hydrophobic non-fluorinated polymeric surfaces for advanced anticorrosive coatings, Polym. Chem.-UK 4 (2013) 926-932.

[16] F. Su, K. Yao, Facile fabrication of superhydrophobic surface with excellent mechanical abrasion and corrosion resistance on copper substrate by a novel method, ACS Appl. Mater. Inter. 6 (2014) 8762-8770.

[17] S. Mousavi, F. Dehghan, E. Saljoughi, S. Hosseini, Preparation of modified polyethersulfone membranes using variation in coagulation bath temperature and addition of hydrophilic surfactant, J. Polym. Res. 19 (2012) 1-12.

[18] Z. SUN, X. REN, K. ZHAO, Z.-G. Zhang, Y.-P. Zhao, L. Chen, Y.-J. Cheng, Advance in prepartion and modification of polyethersulfone nanofiltration membrane, Crit. Rev. Env. Sci. Tec. 35 (2012) 88-92,148.

[19] A. Rahimpour, S.S. Madaeni, Improvement of performance and surface properties of nano-porous polyethersulfone (PES) membrane using hydrophilic monomers as additives in the casting solution, J. Membrane Sci. 360 (2010) 371-379.

[20] M. Laghaei, M. Sadeghi, B. Ghalei, M. Shahrooz, The role of compatibility 
between polymeric matrix and silane coupling agents on the performance of mixed matrix membranes: Polyethersulfone/MCM-41, J. Membrane Sci. 513 (2016) 20-32.

[21] S.-E. Lee, E. Jeong, M.Y. Lee, M.-K. Lee, Y.-S. Lee, Improvement of the mechanical and thermal properties of polyethersulfone-modified epoxy composites, J. Ind. Eng. Chem. 33 (2016) 73-79.

[22] W.J. Chen, J.M. Peng, Y.L. Su, L.L. Zheng, L.J. Wang, Z.Y. Jiang, Separation of oil/water emulsion using Pluronic F127 modified polyethersulfone ultrafiltration membranes, Sep. Purif. Technol. 66 (2009) 591-597.

[23] L. He, W. Li, D. Chen, D. Zhou, G. Lu, J. Yuan, Effects of amino silicone oil modification on properties of ramie fiber and ramie fiber/polypropylene composites, Mater. Design 77 (2015) 142-148.

[24] C.H. Chang, M.H. Hsu, C.J. Weng, W.I. Hung, T.L. Chuang, K.C. Chang, C.W. Peng, Y.C. Yen, J.M. Yeh, 3D-bioprinting approach to fabricate superhydrophobic epoxy/organophilic clay as an advanced anticorrosive coating with the synergistic effect of superhydrophobicity and gas barrier properties, J. Mater. Chem. A 1 (2013) 13869-13877.

[25] Y. Lu, Y.D. Yin, B.T. Mayers, Y.N. Xia, Modifying the surface properties of superparamagnetic iron oxide nanoparticles through a sol-gel approach, Nano Lett. 2 (2002) 183-186.

[26] W.G. Bi, R.J. Song, X.Y. Meng, Z.W. Jiang, S.Y. Li, T. Tang, In situ synthesis of silica gel nanowire/Na+-montmorillonite nanocomposites by the sol-gel route, 
Nanotechnology 18 (2007) 7.

[27] M. Wang, C. Chen, J.P. Ma, J. Xu, Preparation of superhydrophobic cauliflower-like silica nanospheres with tunable water adhesion, J. Mater. Chem. $21(2011) 6962-6967$.

[28] K.M.S. Meera, R.M. Sankar, A. Murali, S.N. Jaisankar, A.B. Mandal, Sol-gel network silica/modified montmorillonite clay hybrid nanocomposites for hydrophobic surface coatings, Colloid Surface B. 90 (2012) 204-210.

[29] A.K. Kota, G. Kwon, A. Tuteja, The design and applications of superomniphobic surfaces, NPG Asia Mater. 6 (2014) 16.

[30] L. Li, P.A. Quinlivan, D.R.U. Knappe, Effects of activated carbon surface chemistry and pore structure on the adsorption of organic contaminants from aqueous solution, Carbon 40 (2002) 2085-2100.

[31] R.M. Mahamood, E.T. Akinlabi, M. Shukla, S. Pityana, Scanning velocity influence on microstructure, microhardness and wear resistance performance of laser deposited Ti6Al4V/TiC composite, Mater. Design 50 (2013) 656-666.

[32] C. Howell, T.L. Vu, J.J. Lin, S. Kolle, N. Juthani, E. Watson, J.C. Weaver, J. Alvarenga, J. Aizenberg, Self-Replenishing Vascularized Fouling-Release Surfaces, ACS Appl. Mater. Inter. 6 (2014) 13299-13307.

[33] H. Jie, Q.J. Xu, L. Wei, Y.L. Min, Etching and heating treatment combined approach for superhydrophobic surface on brass substrates and the consequent corrosion resistance, Corros. Sci. 102 (2016) 251-258.

[34] W. Liu, Q.J. Xu, J. Han, X.H. Chen, Y.L. Min, A novel combination approach for 
the preparation of superhydrophobic surface on copper and the consequent corrosion resistance, Corros. Sci. 110 (2016) 105-113.

[35] R.X. Yuan, S.Q. Wu, B.H. Wang, Z.J. Liu, L.W. Mu, T. Ji, L. Chen, B.W. Liu, H.Y. Wang, J.H. Zhu, Superamphiphobicity and electroactivity enabled dual physical/chemical protections in novel anticorrosive nanocomposite coatings, Polymer 85 (2016) 37-46.

[36] D.W. Zhang, H.C. Qian, L.T. Wang, X.G. Li, Comparison of barrier properties for a superhydrophobic epoxy coating under different simulated corrosion environments, Corros. Sci. 103 (2016) 230-241.

[37] N. Imaz, M. Ostra, M. Vidal, J.A. Diez, M. Sarret, E. Garcia-Lecina, Corrosion behaviour of chromium coatings obtained by direct and reverse pulse plating electrodeposition in $\mathrm{NaCl}$ aqueous solution, Corros. Sci. 78 (2014) 251-259. 


\section{Figure captions}

Fig. 1. Schematic diagrams of the preparation of surperhydrophobic coating. (Inset: the scale bars of SEM are $10 \mu \mathrm{m}$ (a), $10 \mu \mathrm{m}$ (b), $10 \mu \mathrm{m}$ (c) and $20 \mu \mathrm{m}$ (d), respectively.)

Fig. 2. The FT-IR (a) of MMT (i) and the prepared $\mathrm{MMT}_{-} \mathrm{SiO}_{2}$ composite particles (ii), the schematic diagram of nano- $\mathrm{SiO}_{2}$ covered onto MMT surface (b), the morphologies of original MMT (c1, c2), MMT covered by nano- $\mathrm{SiO}_{2}$ with 6 h hydrolysis (d1, d2), and physical mixing with MMT and nano-SiO 2 (e1, e2).

Fig. 3. Influence of the (a) MMT (with constant $0.1 \mathrm{~g}$ APDMS), (b) $\mathrm{MMT}^{-\mathrm{SiO}_{2}}$ (with constant 0.1 g APDMS), (d) APDMS content on the wettabilities of the composite coatings and diagrams illustrate the effect of surface structure on the hydrophobic (c).

Fig. 4. The morphologies of the surfaces under different magnification: PES/APDMS/MMT-12 (a1, a2), PES/APDMS-MMT-SiO ${ }_{2}-6 \quad$ (b1, b2), PES/APDMS-MMT-SiO $2-12$ (c1, c2).

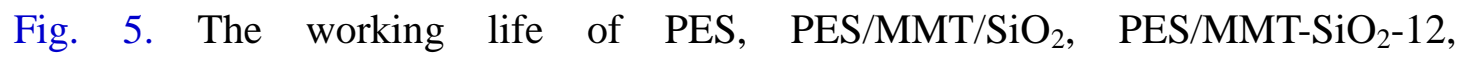
PES/APDMS/MMT-SiO $2-12$ coatings (a), influence of friction process on the wettability of the PES/APDMS/MMT-SiO 2 (b), and the morphologies of the PES/APDMS/MMT-SiO 2 coating after rubbing 1000 cycles (c) and 60000 cycles with rough treated $(\mathrm{d})$.

Fig. 6. Potentiodynamic polarization curves (A) and interface model for anticorrosion mechanism (B) of the untreated aluminum plate (a), covered with PES (b) and the superhydrophobic sample (c) with the immersion time for $1 \mathrm{~d}, 30 \mathrm{~d}, 30 \mathrm{~d}$, 
respectively.

Fig. 7. Impedance spectra of bare Al substrate (a, b, c), pure PES coating (d, e, f) and PES/APDMS/MMT-SiO 2 (g, h, i) composite coating after $1 \mathrm{~d}, 7 \mathrm{~d}, 15 \mathrm{~d}, 30 \mathrm{~d}, 60 \mathrm{~d}$ of immersion tests in $3.5 \mathrm{wt} \% \mathrm{NaCl}$ solution.

\section{Figures}

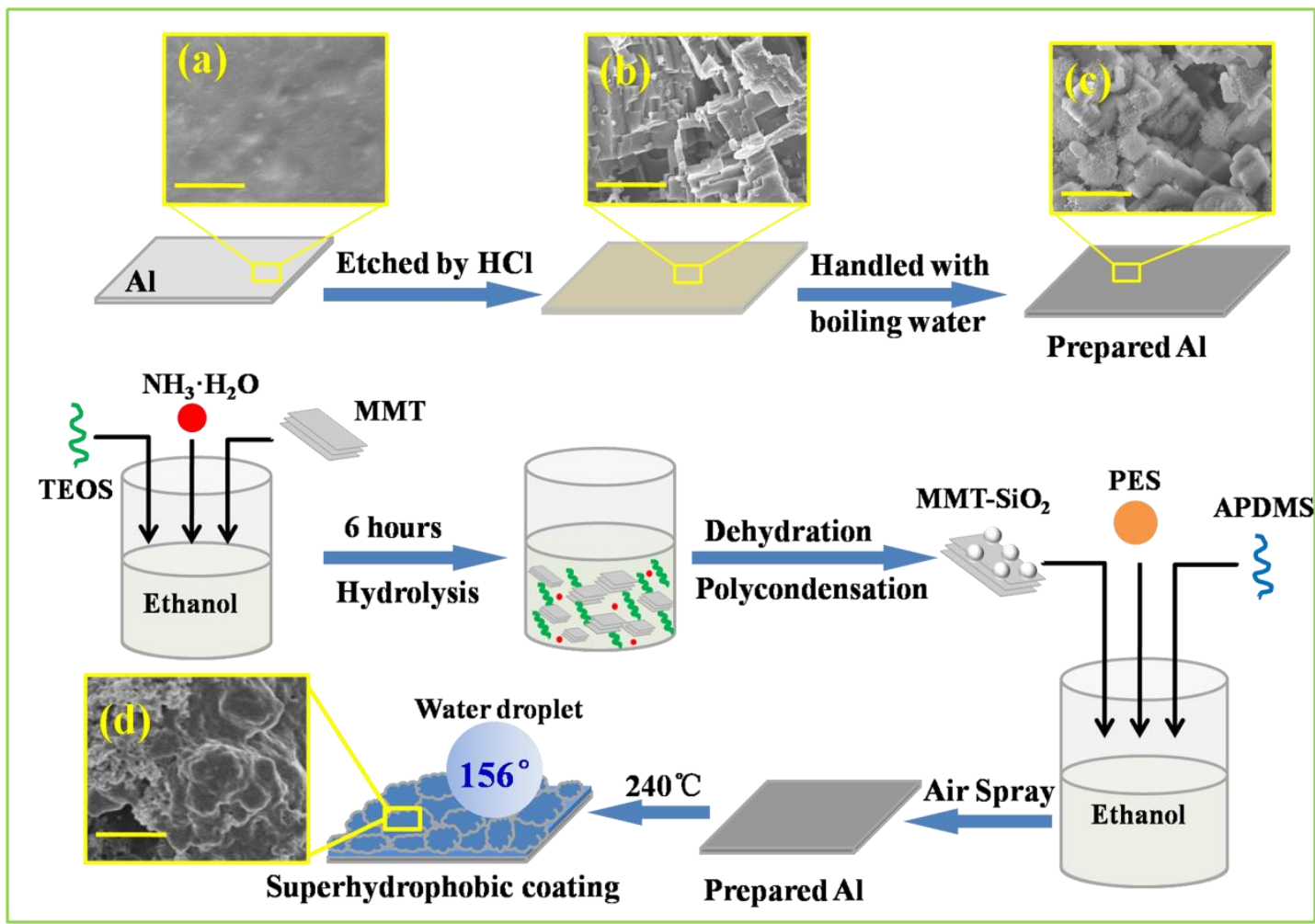

Fig. 1. Schematic diagrams of the preparation of surperhydrophobic coating. (Inset:

the scale bars of SEM are $10 \mu \mathrm{m}$ (a), $10 \mu \mathrm{m}$ (b), $10 \mu \mathrm{m}$ (c) and $20 \mu \mathrm{m}$ (d),

respectively.) 

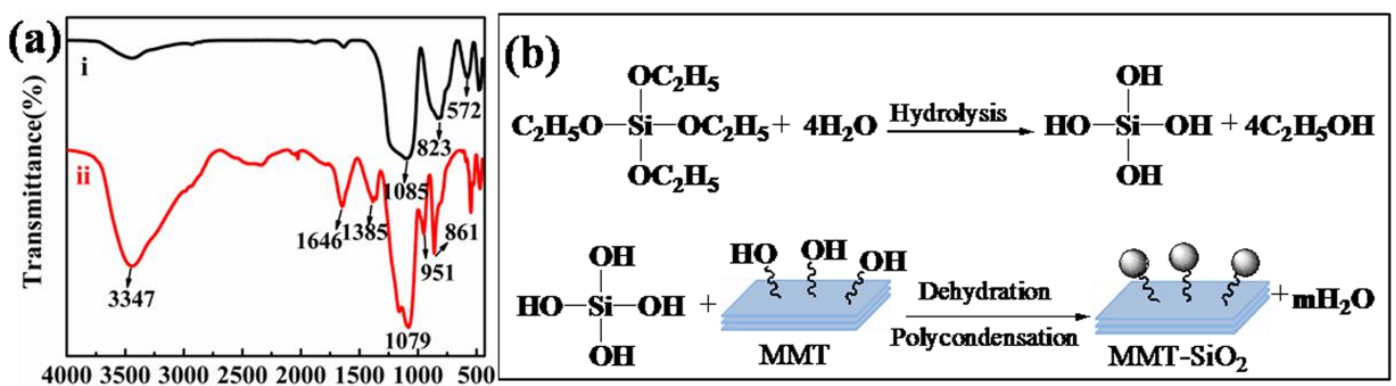

Wavenumbers $\left(\mathrm{cm}^{-1}\right.$,
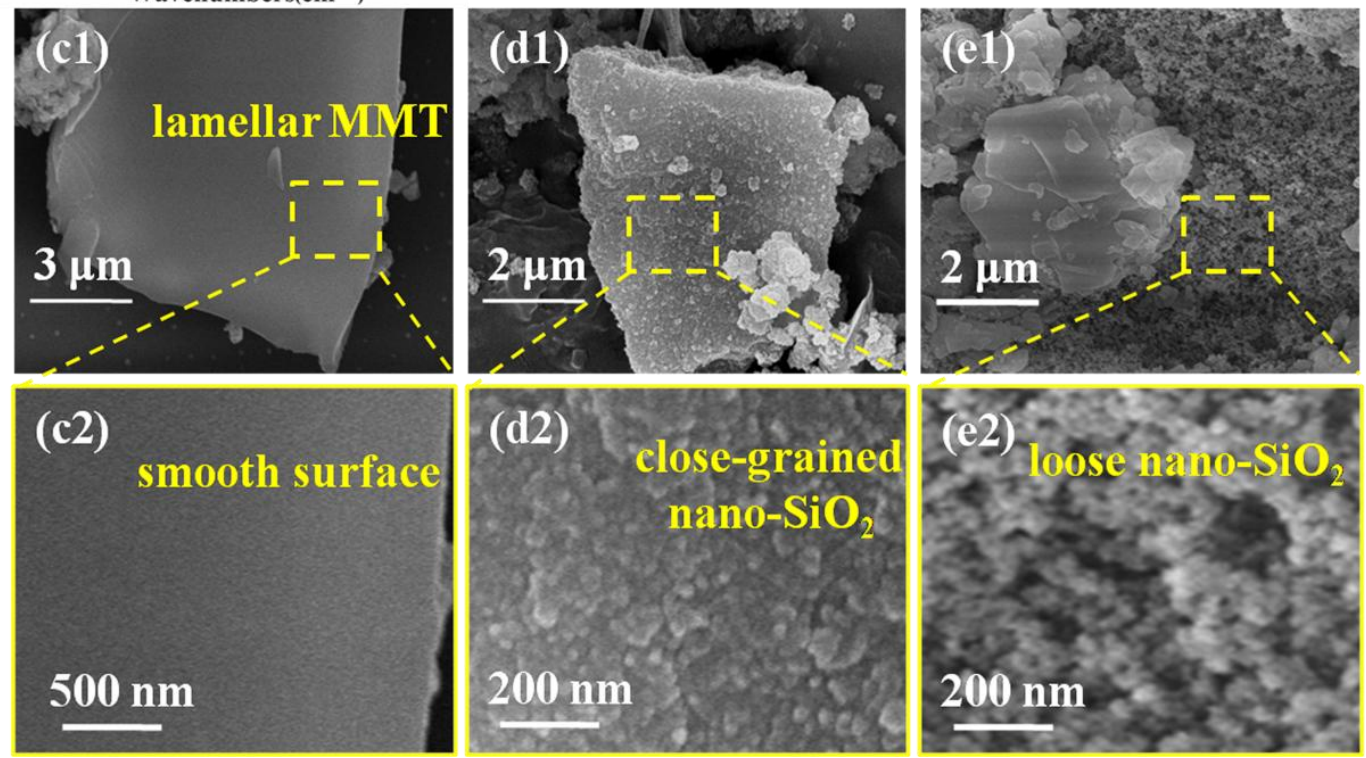

Fig. 2. The FT-IR (a) of MMT (i) and the prepared MMT-SiO 2 composite particles (ii), the schematic diagram of nano- $\mathrm{SiO}_{2}$ covered onto MMT surface (b), the morphologies of original MMT (c1, c2), MMT covered by nano-SiO 2 with 6 h hydrolysis (d1, d2), and physical mixing with MMT and nano- $\mathrm{SiO}_{2}(\mathrm{e} 1, \mathrm{e} 2)$. 

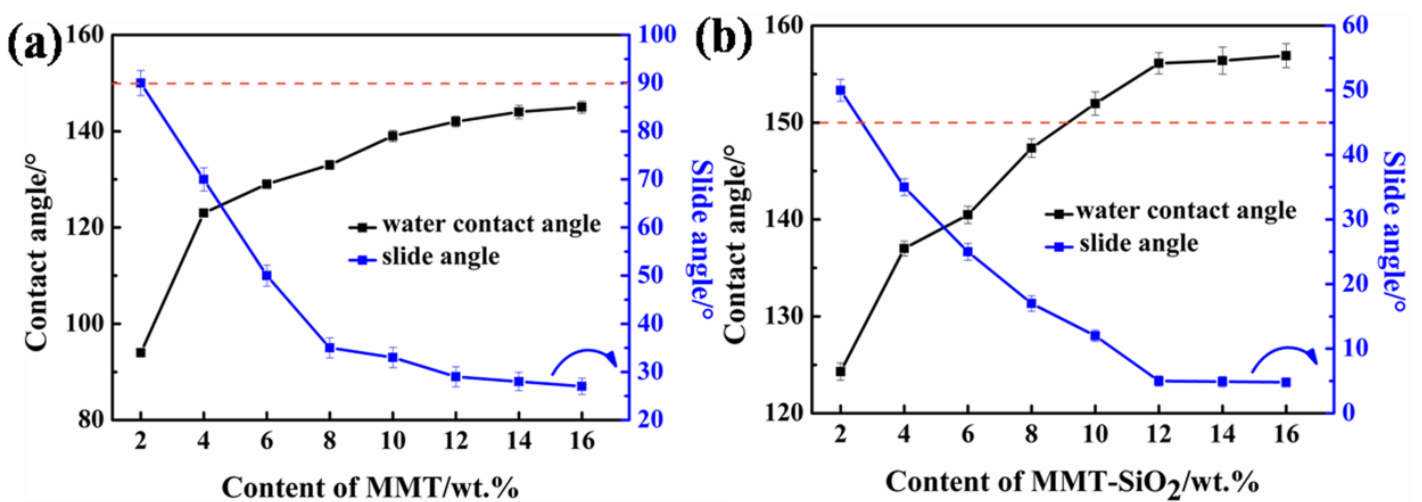

(c)

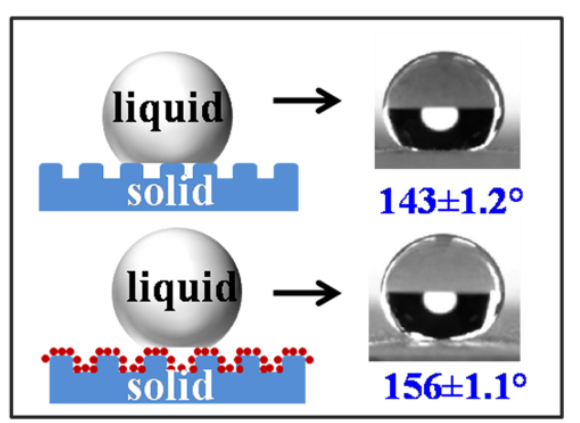

(d)

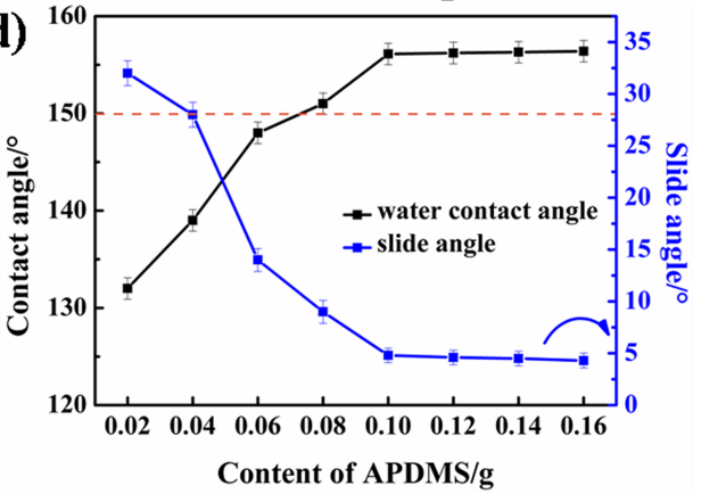

Fig. 3. Influence of the (a) MMT (with constant $0.1 \mathrm{~g}$ APDMS), (b) $\mathrm{MMT}^{-\mathrm{SiO}_{2}}$

(with constant $0.1 \mathrm{~g}$ APDMS), (d) APDMS content on the wettabilities of the composite coatings and diagrams illustrate the effect of surface structure on the hydrophobic (c). 

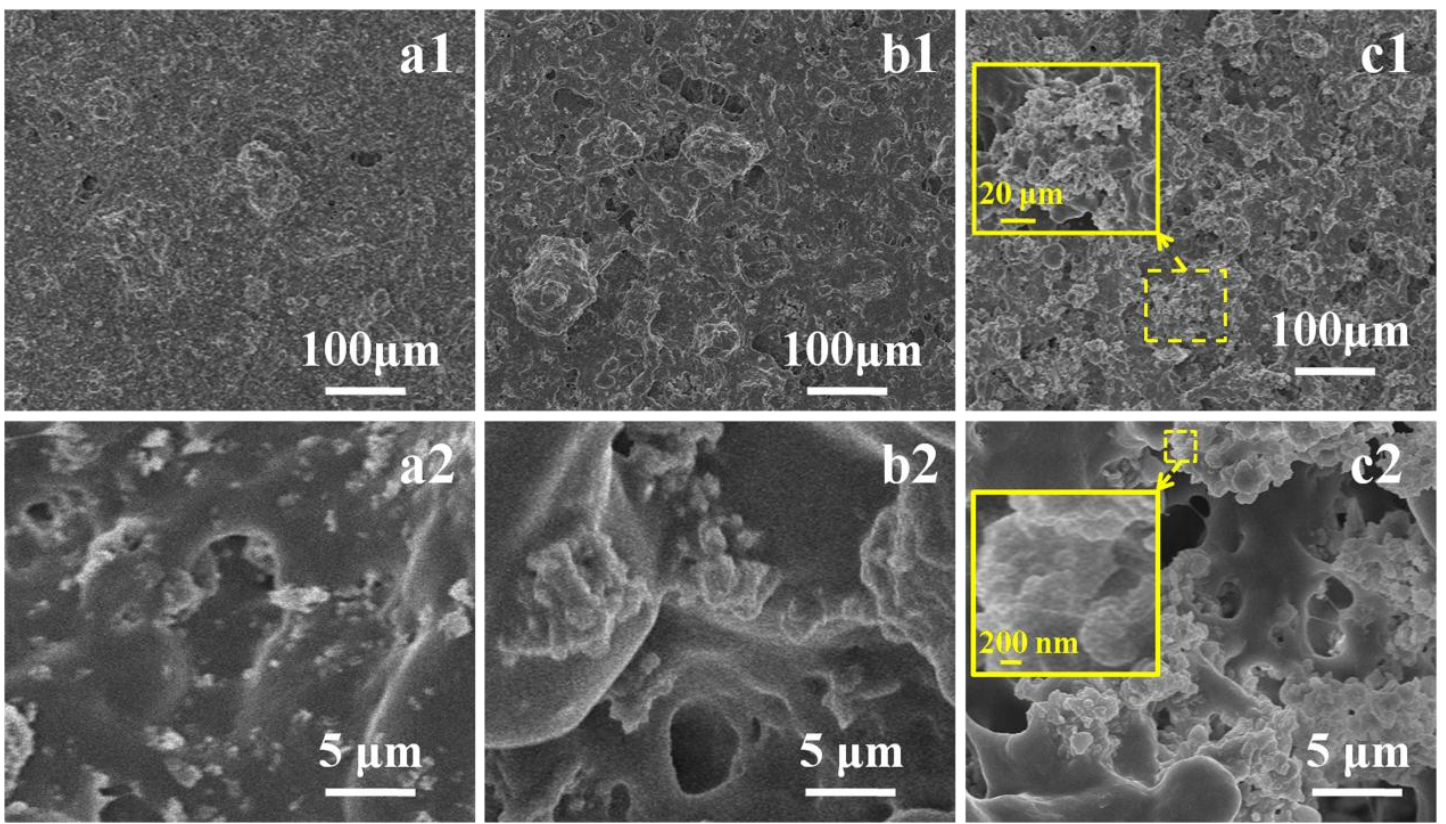

Fig. 4. The morphologies of the surfaces under different magnification:

PES/APDMS/MMT-12 (a1, a2), PES/APDMS-MMT-SiO $2-6$ (b1, b2), PES/APDMS-MMT-SiO $2-12$ (c1, c2). 

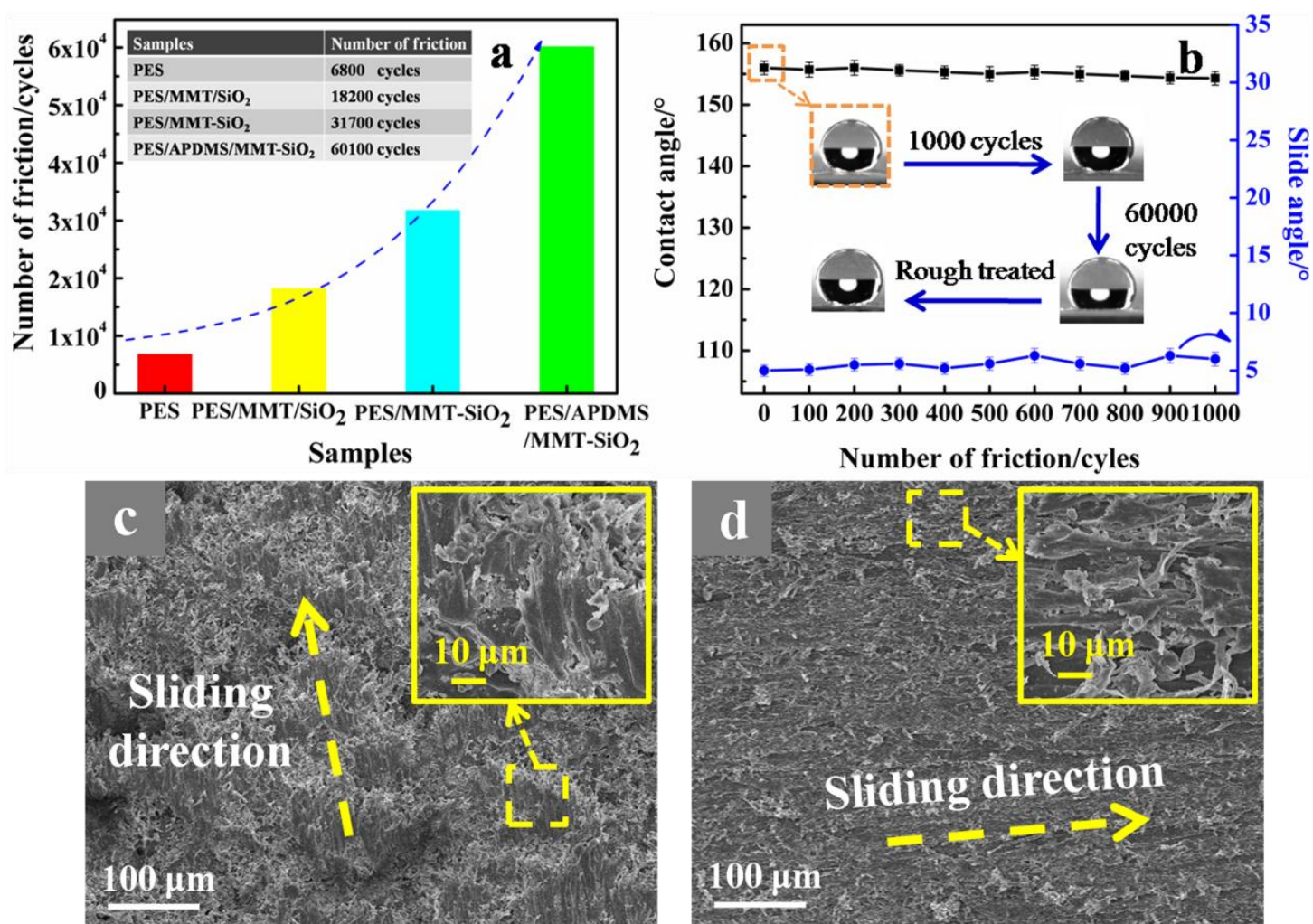

Fig. 5. The working life of PES, PES/MMT/SiO ${ }_{2}, \mathrm{PES} / \mathrm{MMT}-\mathrm{SiO}_{2}-12$,

PES/APDMS/MMT-SiO 2 -12 coatings (a), influence of friction process on the wettability of the PES/APDMS/MMT-SiO ${ }_{2}$ (b), and the morphologies of the

PES/APDMS/MMT-SiO 2 coating after rubbing 1000 cycles (c) and 60000 cycles with rough treated $(d)$. 

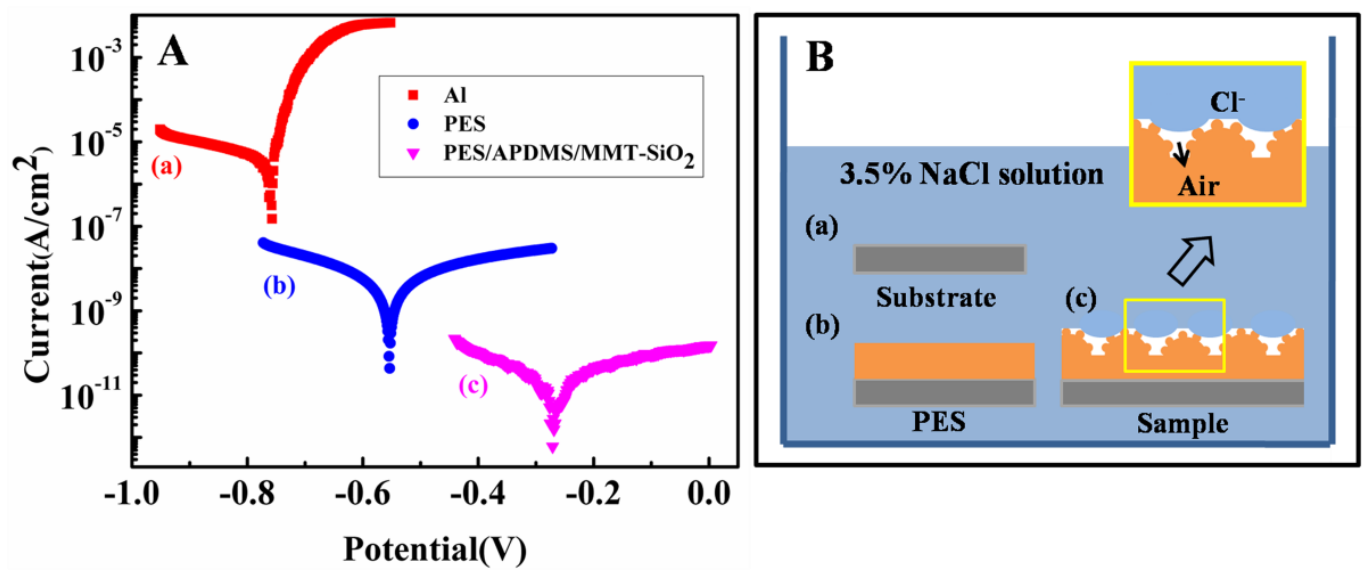

Fig. 6. Potentiodynamic polarization curves (A) and interface model for anticorrosion mechanism (B) of the untreated aluminum plate (a), covered with PES (b) and the superhydrophobic sample (c) with the immersion time for $1 \mathrm{~d}, 30 \mathrm{~d}, 30 \mathrm{~d}$, respectively. 

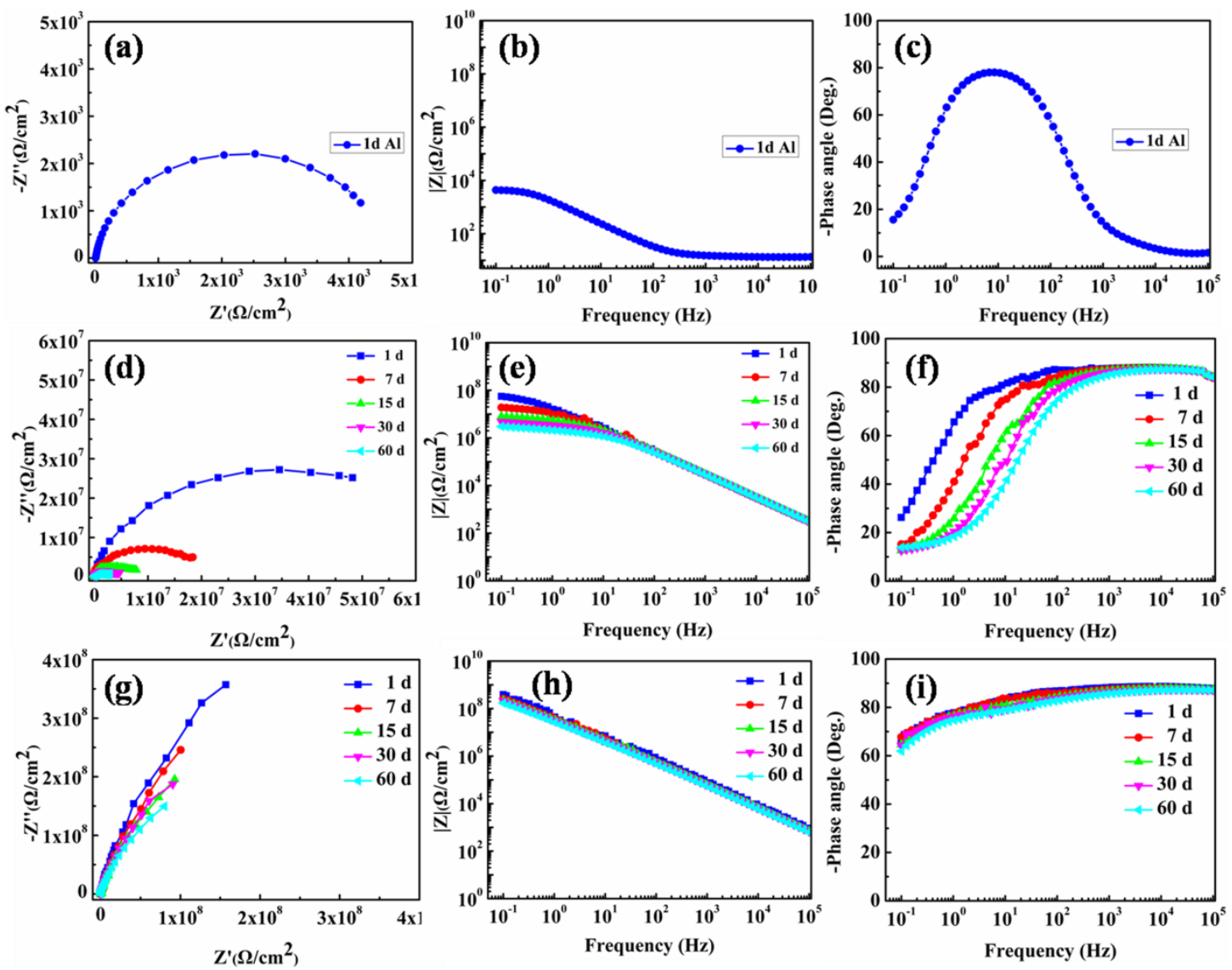

Fig. 7. Impedance spectra of bare Al substrate (a, b, c), pure PES coating (d, e, f) and

PES/APDMS/MMT-SiO 2 (g, h, i) composite coating after $1 \mathrm{~d}, 7 \mathrm{~d}, 15 \mathrm{~d}, 30 \mathrm{~d}, 60 \mathrm{~d}$ of immersion tests in $3.5 \mathrm{wt} \% \mathrm{NaCl}$ solution. 


\section{Fabrication of durable fluorine-free superhydrophobic polyethersulfone (PES) composite coating enhanced by assembled $\mathrm{MMT}^{-\mathrm{SiO}_{2}}$ nanoparticles}

Xiguang Zhang, Huaiyuan Wang*, Zhanjian Liu, Yixing Zhu, Shiqi Wu, Chijia Wang, Yanji Zhu

College of Chemistry and Chemical Engineering, Northeast Petroleum University, Daqing, 163318, Republic of China

\section{Graphical Abstract}

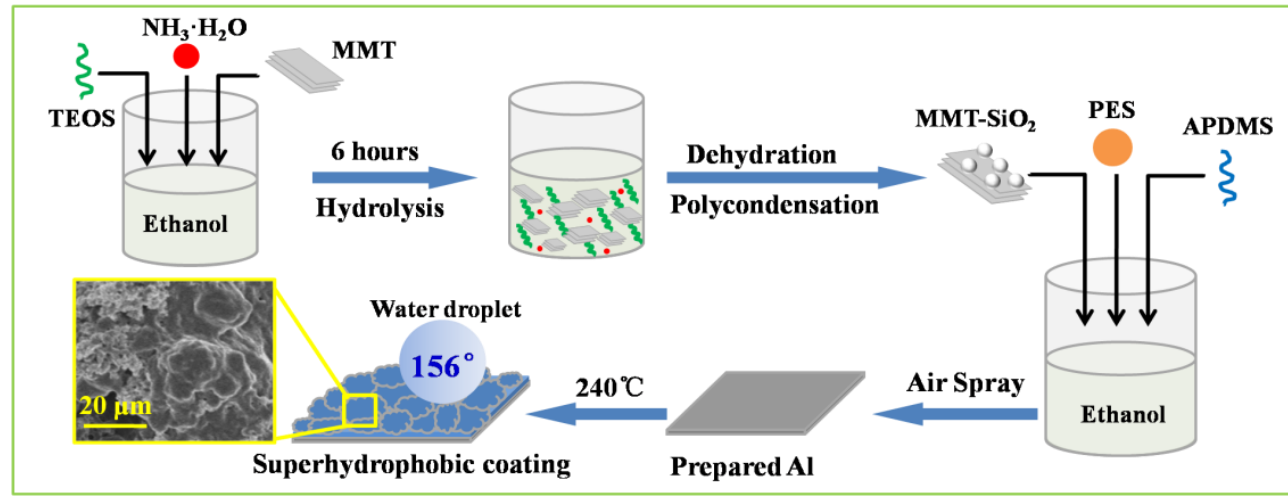

A fluorine-free robust superhydrophobic polyethersulfone (PES) coating has been prepared by combining amino silicon oil (APDMS) and montmorillonite-silica $\left(\mathrm{MMT}^{-\mathrm{SiO}_{2}}\right)$ particles. The MMT-SiO 2 particles were formed by in-situ growth of $\mathrm{SiO}_{2}$ on the MMT surface via sol-gel. The prepared superhydrophobic PES composite coating demonstrated excellent wear and corrosion resistance under rigorous conditions. 